\title{
Postinfektiöse Myasthenia gravis bei einer COVID-19-Patientin
}

\section{Meret Huber ${ }^{a}$ Sophia Rogozinski ${ }^{a}$ Wolfram Puppe ${ }^{b}$ Carsten Framme ${ }^{c}$ Günter Höglinger $^{\mathrm{a}}$ Karsten Hufendiek ${ }^{\mathrm{c}}$ Florian Wegner ${ }^{\mathrm{a}}$}

${ }^{a}$ Klinik für Neurologie, Medizinische Hochschule Hannover, Hannover, Deutschland;

${ }^{b}$ Institut für Virologie, Medizinische Hochschule Hannover, Hannover, Deutschland;

'Klinik für Augenheilkunde, Medizinische Hochschule Hannover, Hannover, Deutschland

\section{Schlüsselwörter}

Anosmie/Ageusie · COVID-19 · Diplopie · neurologische

Manifestation · postinfektiöse Myasthenia gravis · SARS-CoV-2

\section{Zusammenfassung}

Ziel: In der vorliegenden Arbeit berichten wir über den Fall einer jungen Frau mit postinfektiösem Auftreten einer Myasthenia gravis nach einer COVID-19-Infektion mit leichten respiratorischen Symptomen und Anosmie/Ageusie einen Monat vor der Aufnahme in unsere neurologische Abteilung.

Methoden: Die Patientendaten stammten aus den Krankenakten der Medizinischen Hochschule Hannover. Die schriftliche Einverständniserklärung der Patientin lag vor.

Ergebnisse: Die 21-jährige Patientin stellte sich mit subakut aufgetretenen, vertikal versetzten Doppelbildern infolge einer rechtsseitigen partiellen Nervus-okulomotorius-Parese und Ptosis vor. Etwa vier Wochen zuvor waren leichte respiratorische Symptome, Kopfund Gliederschmerzen ohne Fieber sowie eine Anosmie/Ageusie aufgetreten. Bereits während der etwa zehn Tage anhaltenden letztgenannten Symptome hatte die Patientin «müde Augen» und fluktuierende Doppelbilder bemerkt. Die klinische Untersuchung einschließlich eines positiven Tests mit Edrophoniumchlorid und der Nachweis erhöhter Acetylcholinrezeptor-Antikörpern deuteten auf einen ätiologischen Zusammenhang mit der okulären Manifestation einer Myasthenia gravis hin. Mit drei verschiedenen serologischen Tests (Abbott, DiaSorin, Euroimmun) wurden Antikörper (IgA/ lgG) gegen SARS-CoV-2 im Serum nachgewiesen, was für dieses spezifische Coronavirus als Erreger der vorausgegangenen Infektion bei dieser Patientin spricht. Durch Gabe von intravenösen Immunglobulinen und oralem Pyridostigmin wurde das myasthene Syndrom erfolgreich behandelt.

Schlussfolgerung: Dies ist der erste Fallbericht über eine postinfektiöse Myasthenia gravis als neurologische Komplikation bei einer COVID-19-Patientin.

๑) 2020 Huber, Rogozinski, Puppe, Framme, Höglinger, Hufendiek und Wegner

\section{Einführung}

Neben den respiratorischen Symptomen von COVID-19 wurden häufige neurologische Manifestationen darunter auch eine anhaltende Anosmie/Ageusie während der akuten SARS-CoV-2-Infektion beschrieben [1]. Darüber hinaus liegen einige wenige Fallberichte über postinfektiöse neurologische Erkrankungen wie etwa das Guillain-Barré-Syndrom [2-4] und das Miller-FisherSyndrom vor [5].

information@karger.com

(C) 2021 S. Karger GmbH, Freiburg www.karger.com/kop

Karger $\stackrel{\text { ! }}{=}$
In der vorliegenden Arbeit stellen wir den ersten Fall von Myasthenia gravis als neurologische Komplikation nach SARS-CoV2-Infektion vor.

Hufendiek K und Wegner F haben zu gleichen Teilen zur Erstellung dieser Arbeit beigetragen. 


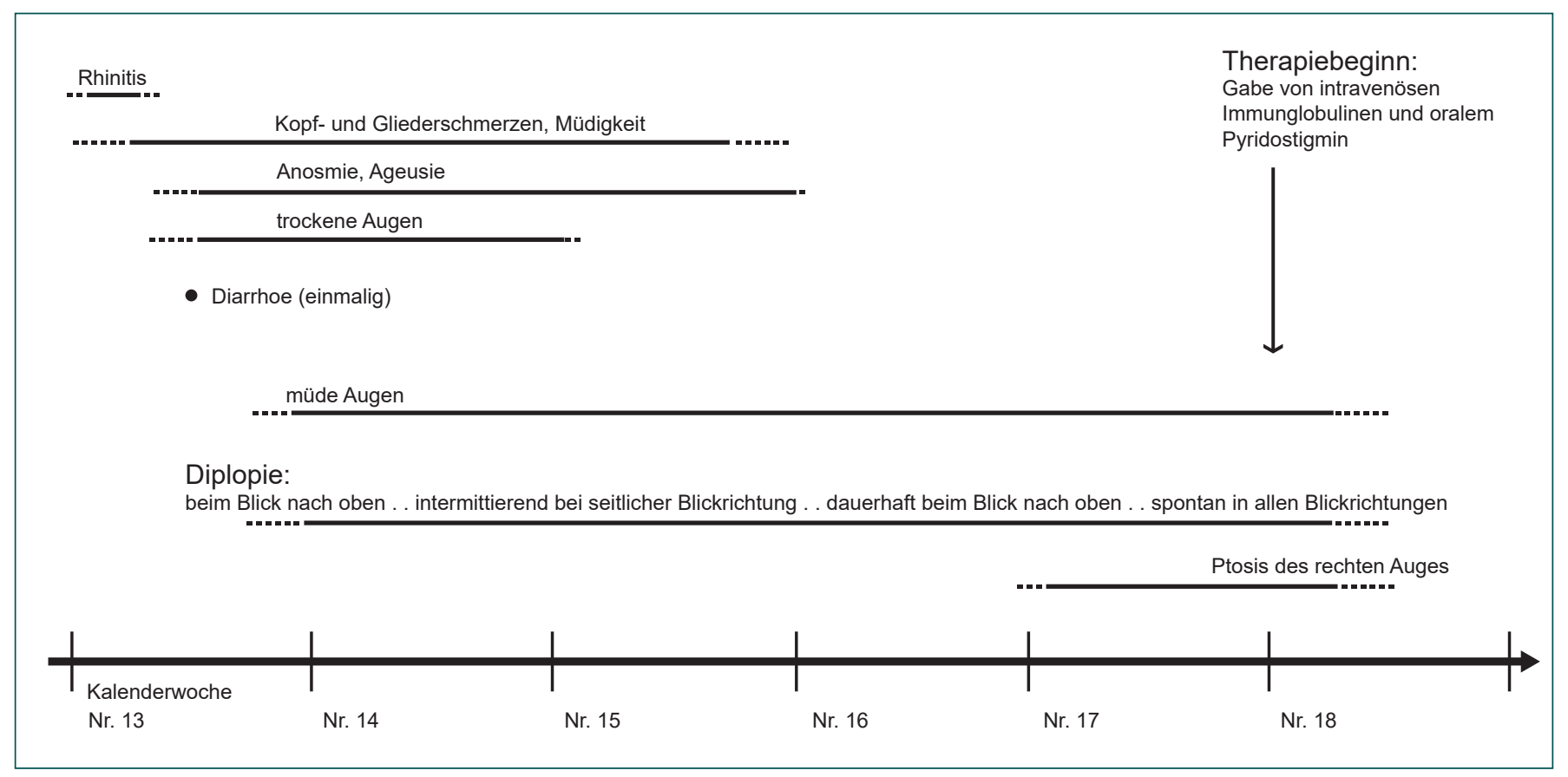

Abb. 1. Zeitlicher Verlauf der COVID-19-Symptome und des überlappenden Auftretens einer okulären Myasthenia gravis, die mit intravenösen Immunglobulinen und oralem Pyridostigmin erfolgreich behandelt wurde.

\section{Methoden}

Euroimmun (Lübeck) stellte SARS-CoV-2-ELISA- (IgA und IgG) und PCR-Tests zur Verfügung. Mithilfe von zwei weiteren ELISAIgG-Tests von Abbott (Sligo, Irland) und DiaSorin (Sasluggia, Italien) wurden die serologischen Ergebnisse validiert.

Eine schriftliche Einverständniserklärung der Patientin (Zustimmung zur Veröffentlichung und Zustimmung zur Veröffentlichung von Fotos/Videos) wurde eingeholt.

\section{Fallbericht}

Eine 21-jährige Frau wurde wegen Diplopie und rechtsseitiger Ptosis, die sich im Verlauf von 5 Tagen verschlimmert hatten, Mitte April 2020 über die Notaufnahme der Medizinischen Hochschule Hannover aufgenommen. Nach Angaben der Patientin waren bei ihr und ihren Familienangehörigen Ende März leichte respiratorische Symptome ohne Fieber, mäßige Müdigkeit mit Kopf- und Gliederschmerzen, trockene Augen- und Nasenschleimhäute sowie Anosmie/Ageusie aufgetreten. Bis April hatten sich alle betroffenen Familienangehörigen erholt, ohne dass ein Test auf SARS-CoV-2 mittels PCR oder Antikörpernachweis erfolgt war.

Die bisherige medizinische Vorgeschichte der Patientin war unauffällig. Zwar sind in der Familie keine neuromuskulären Erkrankungen bekannt, doch gibt es Fälle von Hashimoto-Thyreoiditis, Morbus Addison und perniziöser Anämie. Die drei Geschwister der Patientin sind jedoch gesund, und sie hat keine Kinder.
Die meisten der COVID-19-bedingten Symptome der Patientin hatten sich innerhalb von 3 Wochen zurückgebildet, als postinfektiös okuläre Symptome auftraten (Abb. 1). Initial hatte die Patientin unspezifische visuelle Symptome («müde Augen») bis eine intermittierende, insgesamt jedoch progrediente Diplopie zur Aufnahme führte.

Bei der Mitte April durchgeführten neuroophthalmologischen Untersuchung zeigte sich ein Elevationsdefizit im rechten Auge als Ursache für die vertikalen Doppelbilder und die Ptosis. In der kontrastmittelverstärkten MRT-Untersuchung fanden sich reguläre anatomische Strukturen des Gehirns und der Orbita. Die PCR-Untersuchung eines oropharyngealen Abstrichs, der etwa 4 Wochen nach Beginn der respiratorischen Symptome entnommen wurde, fiel negativ aus, was darauf hindeutete, dass keine akute SARS-CoV-2-Infektion vorlag [6].

Eine zwei Tage später durchgeführte neurologische Untersuchung zeigte eine zunehmende okulomotorische Verschlechterung infolge des Elevationsdefizits und der Ptosis im rechten Auge mit positivem Cogan-Lid-Twitch-Test (Besinger-Score: 7). Bei der Untersuchung beschrieb die Patientin vertikal versetzte Doppelbilder in alle Richtungen mit einem Rückgang der Doppelbilder beim Blick nach links. Der übrige neurologische Status war normal.

Die elektrophysiologischen Untersuchungen ergaben unauffällige motorische und sensorische Messungen ohne Inkrement. Bei repetitiven Stimulationen war kein Dekrement $(3 \mathrm{~Hz})$ des M. orbicularis oculi oder des M. trapezius zu beobachten. Im SimpsonTest zeigte sich eine signifikante Verschlechterung der Ptosis und der Doppelbilder beim forcierten Blick nach oben nach 20 Sekunden. Ein Eisbeuteltest (Ice-on-Eyes-Test) war negativ, wohingegen 


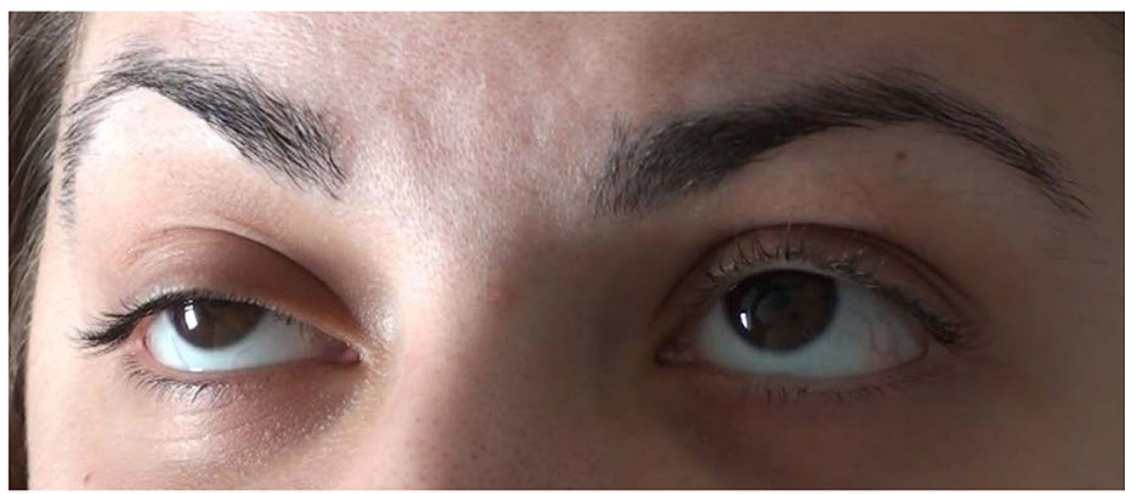

Abb. 2. Überwiegend rechtsseitige Okulomotorius-Parese mit Elevationsdefizit und Ptosis (a), die positiv auf eine intravenöse Testdosis von $9 \mathrm{mg}$ Edrophoniumchlorid ansprach (b), was auf eine okuläre Manifestation einer Myasthenia gravis als Ursache für die Diplopie hindeutet.

B

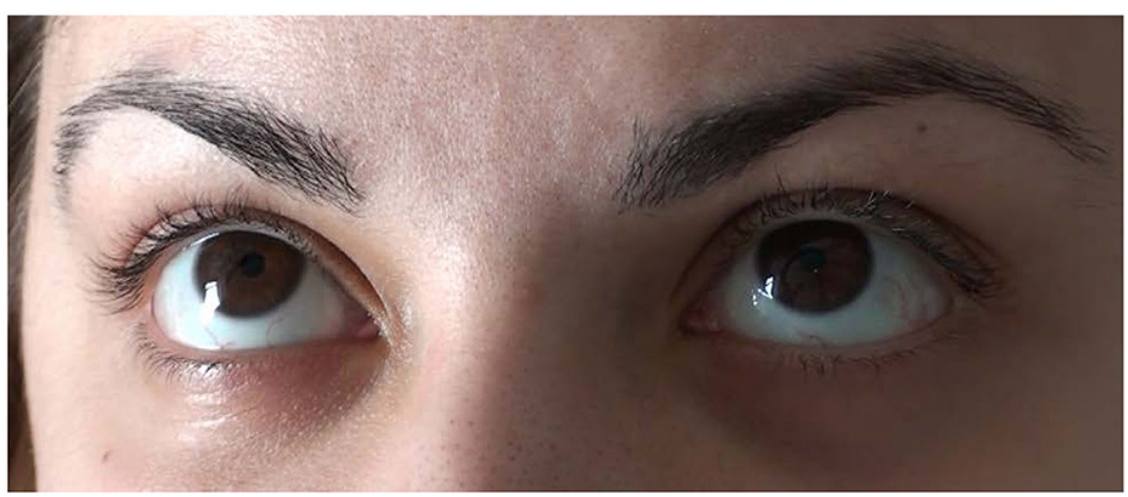

ein Test mit intravenösem Edrophoniumchlorid (mit einer kumulativen Dosis von $9 \mathrm{mg}$ ) eindeutig positiv ausfiel (vollständige Erholung des forcierten Blicks nach oben für 45 Sekunden; Abb. 2). Die Konzentration der Antikörper gegen Acetylcholinrezeptoren im Serum war erhöht $(0,9 \mathrm{nmol} / \mathrm{l}$ anstatt $<0,25 \mathrm{nmol} / \mathrm{l})$. Weitere Blutuntersuchungen einschließlich anderer Myasthenie-assoziierter Antikörper (Agrin, LRP4, MuSK, Titin) und Screening-Parameter für immunologische Erkrankungen einschließlich Gangliosid IgM/IgG fielen unauffällig aus. Die mittels ELISA-Test (Euroimmun) bestimmten SARS-CoV-2-IgA und -IgG-Antikörper im Serum waren positiv und spiegelten die typische COVID19-Vorgeschichte unserer Patientin wider. Zur Validierung der positiven serologischen Ergebnisse für SARS-CoV-2 wurden zwei weitere ELISA-IgG-Tests (Abbott und DiaSorin) durchgeführt; ein IgM-Test stand nicht zur Verfügung. Die Zellzahl im Liquor war in der mikroskopischen Untersuchung normal, ebenso die Protein- und Laktatkonzentration und die Untersuchung auf oligoklonale Banden fiel positiv aus (Typ 2a). Der PCR- und der ELISA-Test auf SARS-CoV-2-IgG/IgA im Liquor waren negativ (Euroimmun, experimentell durchgeführt). Weitere Serum- und Liquoruntersuchungen auf eine Herpesinfektion, Borreliose und Syphilis ergaben keinen Hinweis auf eine akute Infektion.

Es bestanden keine Dysphagie und Dyspnoe und die standardisierten Lungenfunktionstests fielen unauffällig aus. Die Elektroenzephalographie ergab keine auffälligen Befunde. Weitere Untersuchungen mittels Thorax-Röntgenaufnahmen und Magnetresonanztomographie (MRT) zeigten kein Thymom. Das Lungenparenchym wies keine (post)inflammatorischen Veränderungen auf.
Aufgrund der akuten klinischen Exazerbation in den vorangegangenen Tagen erhielt die Patientin intravenös Immunglobuline [7] $(0,4 \mathrm{~g} / \mathrm{kg} / \mathrm{d}$ für 5 Tage $)$ und Pyridostigmin oral $(3 \times 60 \mathrm{mg} / \mathrm{d})$, wodurch es zu einer Rückbildung der okulären Symptome (Besinger-Score 3) vor der Entlassung kam. Nachdem die orale Pyridostigmin-Dosis in den darauf folgenden Wochen schrittweise auf 3 $\times 120 \mathrm{mg} / \mathrm{d}$ erhöht worden war, zeigte sich bei der Nachuntersuchung Mitte Mai eine weitere Verbesserung (Besinger-Score: 0).

\section{Diskussion}

Im vorliegenden Bericht beschreiben wir den Fall einer jungen Frau mit subakuter okulärer Manifestation einer Myasthenia gravis nach einer typischen COVID-19-Infektion einschließlich neurologischer Symptome (Kopfschmerzen, Anosmie/Ageusie). Anosmie/Ageusie sind häufig berichtete neurologische Symptome einer SARS-CoV-2-Infektion [8]. Die meisten COVID-19-Patienten haben keine nasale Obstruktion oder Rhinitis-Symptome, was dafür spricht, dass es sich bei der Anosmie/Ageusie möglicherweise um eine neurologische Komplikation handelt, die durch eine direkte Schädigung der Rezeptoren verursacht wird [8].

Myasthenia gravis ist eine neuromuskuläre Autoimmunerkrankung, die meist durch Antikörper gegen postsynaptische Acetylcholinrezeptoren ausgelöst wird. Allgemein liegen nur wenige neuere Berichte über eine postinfektiöse Myasthenia gravis (nach Varizellen-Infektionen [9, 10]/viraler Pharyngitis [10]) vor. Ein Bericht über eine akute generalisierte schlaffe Lähmung bei einem Kind ohne Liquoranomalien stand im Zusammenhang mit 
einer Coronavirus-Infektion mit HCoV 229E und OC43 [11]. Anders als bei früheren Fällen von postviraler Myasthenia gravis [ 9 , 10] stellten wir bei unserer Patientin erhöhte Acetylcholinrezeptor-Antikörper fest. Ob eine etwaige molekulare Mimikry zwischen dem Acetylcholinrezeptor und den SARS-CoV-2-Proteinen die Immunantwort ausgelöst haben könnte, die zum postinfektiösen Auftreten der Myasthenia gravis geführt hat, ist derzeit noch unklar [12]. Es scheint jedoch keine ausgeprägte strukturelle Übereinstimmung zwischen den relevanten nikotinischen Acetylcholinrezeptor-Untereinheiten (alpha1, beta1, delta, epsilon) und bekannten SARS-CoV-2-Proteinen zu geben (https://www. uniprot.org/blast).

In Kalenderwoche 17, mutmaßlich 4 Wochen nach den initialen respiratorischen Symptomen, wurden bei unserer Patientin IgAund IgG-Antikörper gegen SARS-CoV-2 im Serum nachgewiesen. Dabei kam ein semiquantitativer ELISA IgA/IgG-Test zur Anwendung, der die S1-Domäne nachweist und eine Spezifität von 98,5 \% (für IgG) bzw. 92,5 \% (für IgA) (beide von Euroimmun) besitzt. Die Validierung der serologischen Ergebnisse erfolgte mithilfe von zwei verschiedenen ELISA-IgG-Tests mit einer Spezifität von 98,5\% (DiaSorin) und 99,45\% (Abbott), die $>14$ Tage nach der Infektion durchgeführt wurden. Allerdings sind den Herstellern Kreuzreaktionen mit Cytomegalie-Virus-IgG (Abbott), Hepatitis-B und Influenza A (DiaSorin) oder anderen Coronaviren, insbesondere SARS-CoV-1 (Euroimmun), bekannt. Angesichts der typischen COVID-19-Symptomatik in der medizinischen Vorgeschichte unserer Patientin und der mehrfach positiven serologischen Testergebnisse für SARS-CoV-2 gehen wir davon aus, dass mit hoher Wahrscheinlichkeit eine vorausgegangene Infektion mit dem neuen Coronavirus die Ursache für die postinfektiöse okuläre Manifestation der Myasthenia gravis als neurologische Komplikation war.

Auf der anderen Seite könnte ein COVID-19-Patient auch zufällig an einer überlappenden Myasthenia gravis leiden. Nach unserer Auffassung lässt sich diese Frage auch durch einen retrospektiven Vergleich der Erkrankungsraten nicht vollständig klären. Obwohl die Inzidenz von SARS-CoV-2-Infektionen in unserer Region Ende März 2020 mäßig hoch war (die 7-Tage-Inzidenz betrug nach Angaben des Robert-Koch-Instituts in Niedersachsen 20 bis 28 Neuinfektionen/100 000 Einwohner) und die Myasthenia gravis als seltene Erkrankung gilt (mit einer Inzidenz von 0,25 bis 2/100 000 Einwohner gemäß den Leitlinien der Deutschen Gesellschaft für Neurologie), ist nicht auszuschließen, dass die Myasthenia gravis unabhängig von der SARS-CoV-2-Infektion aufgetreten ist.

Zwar hatte unsere Patientin keine Vorerkrankungen und insbesondere keine neuromuskulären Störungen, doch waren familienanamnestisch Autoimmunerkrankungen bekannt. Dies lässt vermuten, dass COVID-19-Patienten mit ähnlicher Vorgeschichte möglicherweise eine erhöhte Anfälligkeit für postinfektiöse neuroimmunologische Komplikationen durch SARS-CoV-2 aufweisen.

\section{Schlussfolgerung}

In der vorliegenden Arbeit stellen wir den ersten Fall von postinfektiöser Myasthenia gravis im Zusammenhang mit SARS-CoV-2 vor. Es liegen Berichte über eine steigende Zahl von Patienten mit unterschiedlichen neurologischen Symptomen während der akuten Infektion oder postinfektiösen Komplikationen vor, doch sind das vollständige klinische Spektrum sowie die Inzidenz von COVID-19-bedingten neurologischen Manifestationen weiterhin unklar. Zukünftige Studien sollten die Frage aufgreifen, ob eine Assoziation besteht, die höher ist als durch Zufall zu erwarten wäre.

\section{Erklärung zur Datenverfügbarkeit}

Alle für diese Studie generierten Datensätze wurden in den Artikel/das Ergänzungsmaterial eingeschlossen.

\section{Ethikerklärung}

Gemäß den nationalen gesetzlichen Vorschriften und den institutionellen Anforderungen war keine ethische Prüfung und Genehmigung für die Studie mit menschlichen Teilnehmern erforderlich. Die Patienten/Probanden erteilten schriftlich ihre informierte Einwilligung zur Teilnahme an dieser Studie. Es wurde die schriftliche, informierte Zustimmung des/der Probanden für die Veröffentlichung von möglicherweise identifizierbaren Bildern oder Daten, die in diesem Artikel enthalten sind, eingeholt.

\section{Beiträge der einzelnen Autoren}

M. H. und F. W: Konzeption, Organisation und Durchführung des Forschungsprojekts, Erstellung des ersten Entwurfs sowie Überprüfung und kritische Kommentierung des Manuskripts. S. R., W. P., C. F., G. H. und K. H.: Konzeption, Organisation und Durchführung des Forschungsprojekts, Überprüfung und kritische Kommentierung des Manuskripts.

\section{Disclosure Statement}

Die Autoren erklären, dass bei der Durchführung der Forschungsarbeit keine kommerziellen oder finanziellen Beziehungen bestanden, die als potenzieller Interessenkonflikt ausgelegt werden könnten.

\section{Supplementary Material}

Die ergänzenden Materialien zu diesem Artikel können unter $h t t p s: / / w w w$. frontiersin.org/articles/10.3389/fneur.2020.576153/full\#supplementarymaterial abgerufen werden.

\section{Lizenzangabe}

Huber M, Rogozinski S, Puppe W, Framme C, Höglinger G, Hufendiek K, Wegner F: Postinfectious Onset of Myasthenia Gravis in a COVID-19 Patient. Front. Neurol. 2020;11:576153 (DOI: 10.3389/fneur.2020.576153). ${ }^{\circ}$ 2020 Huber, Rogozinski, Puppe, Framme, Höglinger, Hufendiek und Wegner. (Übersetzung), lizensiert unter CC BY 4.0 (https://creativecommons. org/licenses/by/4.0/deed.de).

\section{Literatur}

Die Literatur ist unter www.karger.com/doi/10.1159/000514490 abrufbar. 